\title{
Solitons in the noisy Burgers equation
}

\author{
Hans C. Fogedby and Axel Brandenburg \\ Institute of Physics and Astronomy, University of Aarhus, DK-8000, Aarhus C, Denmark \\ NORDITA, Blegdamsvej 17, DK-2100, Copenhagen Ø, Denmark
}

(October 26, 2018)

\begin{abstract}
We investigate numerically the coupled diffusion-advective type field equations originating from the canonical phase space approach to the noisy Burgers equation or the equivalent Kardar-ParisiZhang equation in one spatial dimension. The equations support stable right hand and left hand solitons and in the low viscosity limit a long-lived soliton pair excitation. We find that two identical pair excitations scatter transparently subject to a size dependent phase shift and that identical solitons scatter on a static soliton transparently without a phase shift. The soliton pair excitation and the scattering configurations are interpreted in terms of growing step and nucleation events in the interface growth profile. In the asymmetrical case the soliton scattering modes are unstable presumably toward multi soliton production and extended diffusive modes, signalling the general non-integrability of the coupled field equations. Finally, we have shown that growing steps perform anomalous random walk with dynamic exponent $z=3 / 2$ and that the nucleation of a tip is stochastically suppressed with respect to plateau formation.
\end{abstract}

PACS numbers: 05.10.Gg, 05.45.-a, 64.60.Ht, 05.45.Yv

\section{INTRODUCTION}

There is a continuing interest in the strong coupling aspects of stochastically driven nonequilibrium systems. The phenomena in question are ubiquitous and comprise turbulence in fluids, interface and growth problems, and chemical and biological systems.

In this context the noisy Burgers equation or the equivalent Kardar-Parisi-Zhang (KPZ) equation, describing the nonequilibrium growth of a noise-driven interface, provide a simple continuum model of an open driven nonlinear system exhibiting scaling and pattern formation.

In one dimension, which is our concern here, the noisy Burgers equation for the local slope, $u(x, t)=\nabla h(x, t)$, of a growing interface has the form [1, 2]

$$
\begin{aligned}
& \frac{\partial u}{\partial t}=\nu \nabla^{2} u+\lambda u \nabla u+\nabla \eta, \\
& \langle\eta(x t) \eta(00)\rangle=\Delta \delta(x) \delta(t) .
\end{aligned}
$$

The height profile (in a comoving frame) $h(x, t)$ is then governed by the equivalent KPZ equation [3, 3 ]

$$
\frac{\partial h}{\partial t}=\nu \nabla^{2} h+\frac{\lambda}{2}(\nabla h)^{2}+\eta .
$$

In (1) and (3) $\nu$ is the damping or viscosity characterizing the linear diffusive term, $\lambda$ a coupling strength for the nonlinear mode coupling or growth term, and $\eta$ a Gaussian white noise, driving the system into a statistically stationary state. The noise is correlated according to (2) and characterized by the strength $\Delta$. Moreover, the Burgers equation is invariant under the slope-dependent Galilean transformation

$$
x \rightarrow x-\lambda u_{0} t, \quad u \rightarrow u+u_{0},
$$

i.e., the interface is superimposed with a constant slope in a moving frame.

The Burgers equation (1) and its KPZ equivalent in one and higher dimensions have been the subject of intense scrutiny in recent years owing to its paradigmatic significance within the field theory of nonequilibrium systems $\left[\begin{array}{l}515 \\ 15\end{array}\right.$

In a series of papers the one dimensional case defined by (11) and (22) has been analyzed in an attempt to uncover the physical mechanisms underlying the pattern formation and scaling behavior. Emphasizing that the noise strength $\Delta$ constitutes the relevant nonperturbative parameter that is driving the system into a statistically stationary state, the method was initially based on a weak noise saddle point approximation to the MartinSiggia-Rose functional formulation of the noisy Burgers equation 16 19]. This work was a continuation of earlier work based on the mapping of a solid-on-solid model onto a continuum spin model [20]. More recently the functional approach has been superseded by a canonical phase space method deriving from the canonical structure of the Fokker-Planck equation associated with the Burgers equation 21 25. Below we briefly summarize these findings.

The functional or the equivalent phase space approach valid in the weak noise limit, $\Delta \rightarrow 0$, replaces the stochastic Langevin-type Burgers equation (11) by coupled deterministic diffusion-advection type mean field equations,

$$
\begin{aligned}
& \frac{\partial u}{\partial t}=\nu \nabla^{2} u-\nabla^{2} p+\lambda u \nabla u, \\
& \frac{\partial p}{\partial t}=-\nu \nabla^{2} p+\lambda u \nabla p,
\end{aligned}
$$

for the slope $u(x, t)$ and a canonically conjugate noise field $p(x, t)$, replacing the stochastic noise $\eta$. The field equations bear the same relation to the Fokker-Planck 
equations as the classical equations of motion bear to the Schrödinger equation in the semi-classical WKB approximation [26].

To justify the weak noise limit we recall the analogy with the WKB approximation in quantum mechanics which, owing to its nonperturbative character, captures features like bound states and tunneling amplitudes, which are generally inaccessible to perturbation theory. Therefore, we anticipate that the present weak noise approach to the Burgers equation also accounts correctly, at least in a qualitative sense, for the stochastic properties even at larger noise strength. However, there may be an upper threshold value beyond which the system may enter a new stochastic or kinetic phase. In the one dimensional case discussed here the scaling behavior is controlled by a single strong coupling fixed point which can be accessed by the present weak noise approach. In two and higher dimension a dynamic renormalization group analysis predicts a kinetic phase transition at a critical noise strength (or coupling strength) and the weak noise approach presumably fails.

The equations (5) and (6) derive from a principle of least action characterized by an action $S\left(u^{\prime} \rightarrow u^{\prime \prime}, t\right)$ associated with an orbit $u^{\prime}(x) \rightarrow u^{\prime \prime}(x)$ traversed in time $t$ [27,

$$
S\left(u^{\prime} \rightarrow u^{\prime \prime}, t\right)=\int_{0, u^{\prime}}^{t, u^{\prime \prime}} d t d x\left(p \frac{\partial u}{\partial t}-\mathcal{H}\right),
$$

with Hamiltonian density

$$
\mathcal{H}=p\left(\nu \nabla^{2} u+\lambda u \nabla u-\frac{1}{2} \nabla^{2} p\right) .
$$

The action is of central importance in the present approach and serves as a weight function for the noisedriven nonequilibrium configurations in much the same manner as the energy $E$ in the Boltzmann factor $\exp (-\beta E)$ for equilibrium systems, where $\beta$ is the inverse temperature. The dynamical action in fact replaces the energy in the context of the dynamics of stochastic nonequilibrium systems governed by a generic Langevin equation driven by Gaussian white noise. The action provides a methodological approach and yields access to the time dependent and stationary probability distributions,

$$
\begin{aligned}
& P\left(u^{\prime} \rightarrow u^{\prime \prime}, t\right) \propto \exp \left[-\frac{S\left(u^{\prime} \rightarrow u^{\prime \prime}, t\right)}{\Delta}\right], \\
& P_{\mathrm{st}}\left(u^{\prime \prime}\right)=\lim _{t \rightarrow \infty} P\left(u^{\prime} \rightarrow u^{\prime \prime}, t\right)
\end{aligned}
$$

and associated moments, e.g., the stationary slope correlations

$$
\begin{aligned}
& \langle u(x t) u(00)\rangle= \\
& \int \prod d u u^{\prime \prime}(x) u^{\prime}(0) P\left(u^{\prime} \rightarrow u^{\prime \prime}, t\right) P_{\mathrm{st}}\left(u^{\prime}\right) .
\end{aligned}
$$

The canonical formulation associates the conserved energy $E$ (following from time translation invariance), the conserved momentum $\Pi$ (from space translation invariance), and the conserved area $M$ (from the Burgers equation with conserved noise):

$$
\begin{aligned}
& E=\int d x \mathcal{H}, \\
& \Pi=\int d x u \nabla p, \\
& M=\int d x u .
\end{aligned}
$$

The field equations (5) and (6) determine orbits in a canonical up phase space where the dynamical issue in determining $S$ and thus $P$ is to find an orbit from $u^{\prime}$ to $u^{\prime \prime}$ in time $t, p$ being a slaved variable. Note that unlike dynamical system theory we are not considering the asymptotic properties of a given orbit. In general the orbits in phase space lie on the manifolds determined by the constants of motion $E, \Pi$ and $M$. Here the zero energy manifold $E=0$ plays a special role in defining the stationary state. For vanishing or periodic boundary conditions for the slope field the zero energy manifold is composed of the transient submanifolds $p=0$ and the stationary submanifold $p=2 \nu u$. The zero energy orbits on the $p=0$ manifold correspond to solutions of the damped noiseless Burgers equation; the orbits on the $p=2 \nu u$ are solutions to the undamped noiseless Burgers equation with negative damping, i.e., $\nu$ replaced by $-\nu$. In the solvable linear case of the noise driven diffusion equation for $\lambda=0$, i.e., the Edwards-Wilkinson equation 281, a finite energy orbit from $u^{\prime} \rightarrow u^{\prime \prime}$ in time $t$ migrates to the zero energy manifold in the limit $t \rightarrow \infty$, yielding according to Eqs. (17) and (10) the stationary distribution $P_{\text {st }} \propto \exp \left(-(\nu / \Delta) \int d x u^{2}\right)$. This distribution also holds in the Burgers case and is a generic result independent of $\lambda[29$. Finally, in the long time limit an orbit from $u^{\prime} \rightarrow u^{\prime \prime}$ is attracted to the hyperbolic saddle point at the origin in phase space implying ergodic behavior in the stationary state. In Fig. 1 we have schematically depicted possible orbits in phase space.

The field equations (5) and (6) admit nonlinear soliton or smoothed shock wave solutions which are, in the static case, of kink-like form,

$$
u_{1}(x)=u \tanh \left[\frac{\lambda|u|}{2 \nu} x\right]
$$

Propagating solitons are subsequently generated by the Galilean boost (4). Denoting the right and left boundary values by $u_{+}$and $u_{-}$, respectively, the propagation velocity is given by

$$
u_{+}+u_{-}=-2 v / \lambda
$$




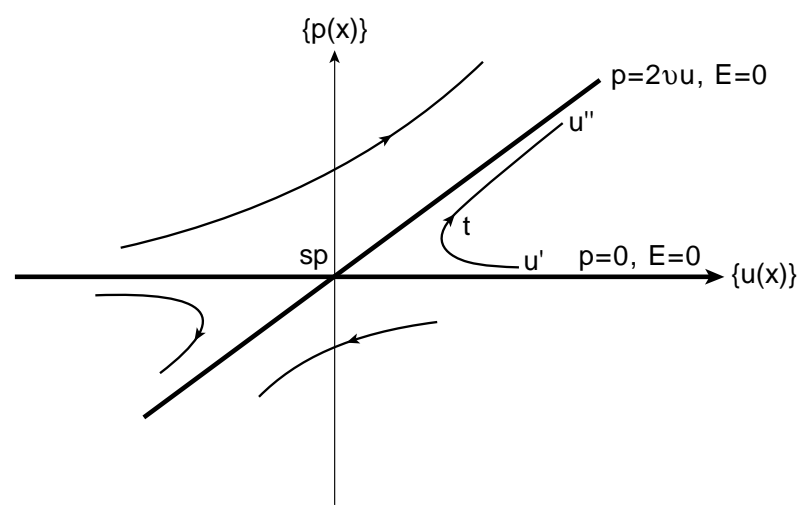

FIG. 1. Generic behavior of the orbits in up phase space. Heavy lines indicate the zero energy manifold. The stationary saddle point (sp) is at the origin. The finite time orbit from $u^{\prime}$ to $u^{\prime \prime}$ is attracted to the saddle point for $t \rightarrow \infty$.

The amplitude of the static soliton is $u$ and the soliton is located at the origin. The right hand soliton for $u>0$, i.e., the soliton with the larger right hand side boundary value, moves on the noiseless manifold $p=0$ and is also a solution of the damped (stable) noiseless Burgers equation for $\eta=0$. The noise-induced left hand soliton for $u<0$, i.e., the soliton with the larger left hand side boundary value, is associated with the noisy manifold $p=2 \nu u$, and is a solution of the undamped (unstable) noiseless Burgers equation with $\nu$ replaced by $-\nu$. In addition the field equations also admit linear mode solutions superimposed as ripple modes on the solitons [30]. The ripple modes are superpositions of both decaying and growing components reflecting the noiseless and noisy manifolds $p=0$ and $p=2 \nu u$, respectively. The soliton mode induces a propagating component with velocity $\lambda u$ in such a way that the right hand soliton acts like a sink and the left hand soliton as a source of linear modes. In the Edwards-Wilkinson limit for $\lambda \rightarrow 0$ the ripple modes become the usual diffusive modes (growing and decaying) of the driven stationary diffusion equation.

The heuristic physical picture that emerges from our analysis is that of a many body formulation of the pattern formation of a growing interface in terms of a dilute gas of propagating solitons matched according to the soliton condition (16) with superimposed linear ripple modes. In Fig. 2 we have depicted the basic soliton modes constituting the building blocks in the representation of a growing interface. For further illustration we have shown in Fig. 3 the slope field $u$, the corresponding height field $h$, and the noise field $p$ for a 4 -soliton configuration.

In the present paper we embark on a numerical analysis of the coupled field equations (5) and (6) with the purpose of investigating them in more detail and provide a numerical underpinning of the heuristic quasi-particle picture advanced in the work referred to above. The paper is organized in the following manner. In Section 2 we discuss the soliton modes. In Section 3 we introduce the numerical method designed in order to treat the inherent instability. In Section 4 we present our numerical results for the scattering of two single solitons on a static soliton and the scattering of two soliton pairs. In Section 5 we discuss growth and nucleation associated with the modes investigated numerically. Section 6 is devoted to a summary of our results and a conclusion.
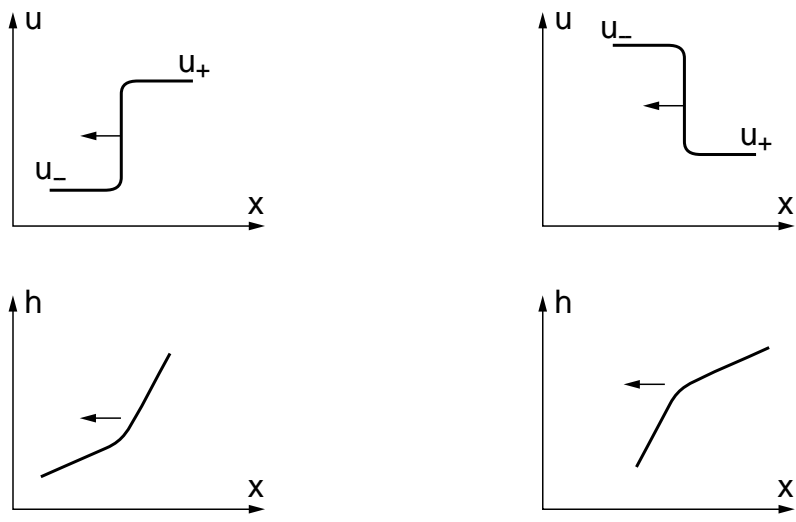

FIG. 2. Slope field $u$ and height profile $h$ for the right hand and left hand moving kink solitons forming the 'quarks' in the description of a growing interface.
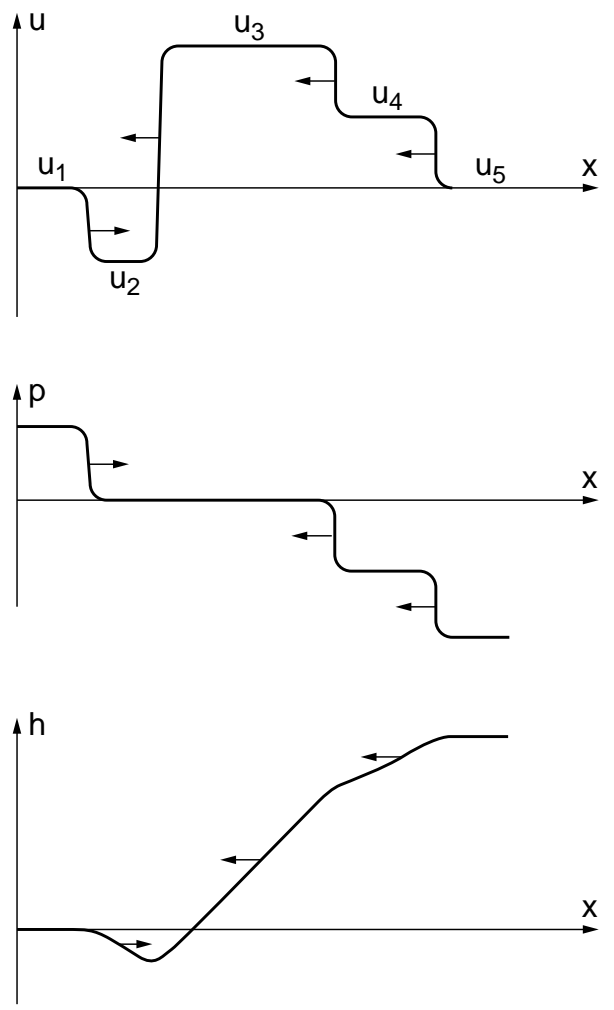

FIG. 3. 4-soliton representation of the slope field $u$, the noise field $p$, and the height field $h$. 


\section{SOLITON MODES}

The exact right and left hand soliton solutions of the field equations do not satisfy periodic or vanishing boundary conditions in the slope field $u$; the nonvanishing boundary values $u_{+}$and $u_{-}$in fact correspond to a deterministic current dissipated or generated at the soliton centers yielding permanent profile solutions [31]. The kink solitons constitute the elementary building blocks or 'quarks' in the present approach and the interface profile is then built up by matching solitons according to the matching condition (16).

The simplest mode satisfying periodic boundary conditions is the two-soliton or pair soliton configuration

$$
u_{2}(x, t)=u_{1}\left(x-v t-x_{1}\right)-u_{1}\left(x-v t-x_{2}\right),
$$

obtained by matching a right hand and a left hand soliton boosted to the velocity $v=-\lambda u$. The two-soliton mode has amplitude $2 u$ and size $\left|x_{2}-x_{1}\right|$. The associated noise field vanishes for the right hand component and equals $2 \nu u$ for the left hand component; we thus have

$$
\begin{aligned}
& p_{2}(x, t)=-2 \nu u_{1}\left(x-v t-x_{2}\right) \text { for } u>0 \\
& p_{2}(x, t)=+2 \nu u_{1}\left(x-v t-x_{1}\right) \text { for } u<0 .
\end{aligned}
$$

By inspection it is seen that the pair mode $(\sqrt{17})$ is an approximate solution to the field equations (5) and (6). The correction terms are of the type $u \nabla u$ and $u \nabla p$ referring to the distinct components of $u_{2}$ and $p_{2}$ and thus correspond to local perturbations from a region of size $\nu / \lambda|u|$ which is small in the low viscosity limit $\nu \rightarrow 0$. We assume that the correction can be treated within a linear stability analysis and thus gives rise to a linear mode propagating between the right hand and left hand solitons 30.

The pair mode thus forms a long-lived excitation or quasi-particle in the many body description of a growing interface. Subject to periodic boundary conditions this mode corresponds to a simple growth situation. The propagation of the pair mode corresponds to the propagation of a step in the height field $h$. At each revolution of the pair mode the interface grows by a uniform layer of thickness $2 u\left|x_{2}-x_{1}\right|$. In Fig. 4 we have depicted the pair mode in $u$, the associated noise field $p$, and the height profile $h$.

Generally a growing interface, ignoring the superimposed linear ripple modes, can at a given time instant be represented by a gas of matched left hand and right hand solitons as depicted in Fig. 3 in the 4-soliton case. A gas of pair solitons thus constitute a particular growth mode where the height profile between moving steps has horizontal segments.
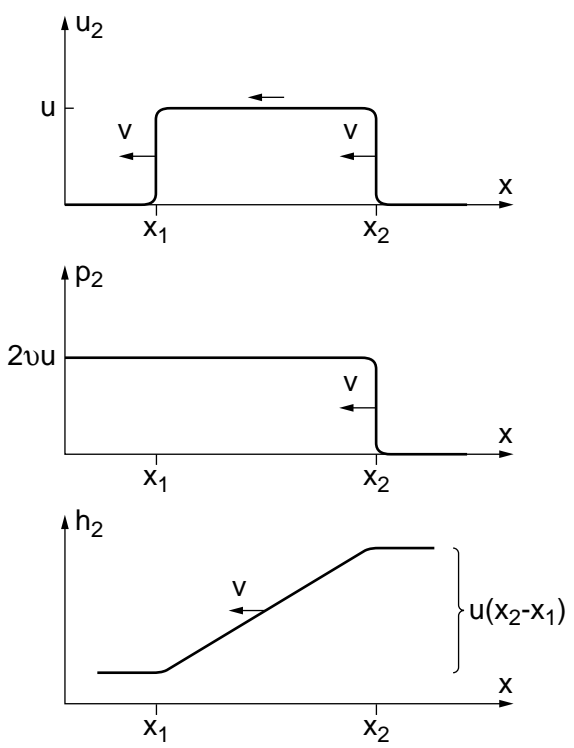

FIG. 4. Slope field $u_{2}$, the associated noise field $p_{2}$, and the resulting height profile $h_{2}$ at time $t=0$ for a two-soliton configuration.

The soliton picture also allows us easily to understand in what sense the right hand soliton acts like a drain and the left hand soliton as a source with respect to perturbations. Considering two pair solitons superimposed on the right and left horizontal parts of the static solitons (15) it follows from (16) that for a right hand soliton perturbations move toward the soliton center and for a left hand soliton perturbations move away from the soliton center. This mechanism also follows from the linear analysis of ripple modes in [30]. The mechanism is depicted in Fig. 5

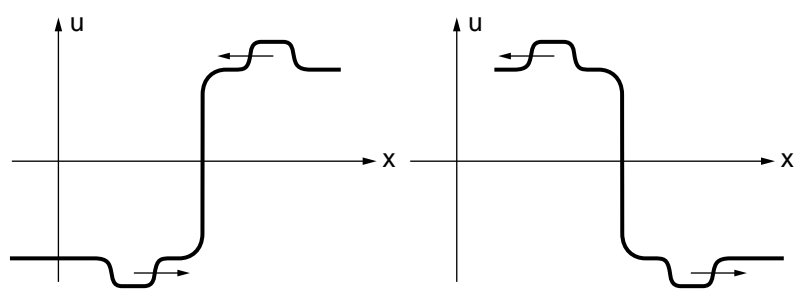

FIG. 5. Source and drain mechanism for the right hand and left hand solitons. The perturbation attracted and repelled by the soliton centers are modeled by pair solitons.

Since we do not posses explicit propagating multisoliton solutions of the field equations (5) and (6) the problem of soliton collisions remains unresolved from an analytical point of view. Therefore we now turn to a numerical analysis of the problem. 


\section{NUMERICAL METHOD}

The coupled field equations (5) and (5) are of the diffusion-advection type with the exception that the evolution of $p$ is governed by a negative diffusion coefficient. Standard numerical methods designed to step the equations forward in time fail because small perturbations with wavenumber $k$ grow in time like $\exp \left(\nu k^{2} t\right)$, so perturbations with the largest possible $k$ grow fastest, rendering hence the integration unstable. In order to circumvent this problem we have designed a method to solve the equations iteratively starting with a trial solution for $u$ and $p$ in the space interval $|x| \leq L$ and time interval $0 \leq t \leq T$. At each iteration step we proceed in two sweeps. In the forward sweep we step only the equation for $u$ forward in time using $p(x, t)$ from the previous iteration step. In the backward sweep we step the equation for $p$ backward in time, using $u(x, t)$ from the forward sweep. In this manner the unwanted perturbations in $p$ decrease exponentially as one moves backward in time.

A drawback of this method is that we can specify initial values only for $u$, and that we must instead specify $p$ at the final time $t=T$. In the present context where we want to consider collisions of solitons this is not a serious problem, because here it is possible to guess the final solution. The numerical solution serves therefore mainly as a tool to check that a certain guess is actually a solution. Furthermore, this method allows us to calculate the precise functional form of $u$ and $p$ during the collision, even if the initial guess around the time of the collision was actually wrong.

We have solved the equations on a mesh with $N_{x}=$ 1001 mesh points and $N_{t}=6001$ time steps. For $L=1 / 2$ we have a mesh spacing of $\Delta x=0.001$. For both sweeps we use sixth order finite differences to calculate first and second derivatives and a third order Runge-Kutta scheme for the time integration (see, e.g., the appendix of Ref. 32 for these schemes).

In order to adequately resolve $u$ and $p$ at all times we must choose a suitable value of $\nu$. We found empirically that $\nu=0.005$ gave good results, which is the value adopted in the following. For smaller values of $\nu$ the $u$ and $p$ functions become only marginally resolved whereas for larger values of $\nu$ the length of the time step is mostly controlled by the value of $\nu$ rather than just the propagation speeds of the solitons. Empirically we found that the maximum time step that can be used is $\Delta t=5 \times 10^{-5}$ for $\nu=0.005$. In all cases we have chosen $\lambda=1$.

\section{SOLITONS, PAIR SOLITONS, AND SOLITON COLLISIONS}

In choosing soliton configurations to be verified by the numerical method we have found that it is essential to satisfy the three conservation laws governing the dynamics of solitons, namely the conservation of energy (12), the conservation of momentum (13), and the conservation of area (14).

\section{A. Solitons and pair soliton}

We have numerically verified that the right and left hand solitons (15) for $u>0$ and $u<0$ are solutions. By construction the two-soliton configuration (17) carries energy $E_{2}=(-16 / 3) \lambda \nu|u|^{3}$, momentum $\Pi_{2}=-4 \nu u|u|$, and for small $\nu$ the area $M_{2} \propto 2 \nu\left|x_{1}-x_{2}\right|$. We have shown that, for small $\nu$, a well-separated two-soliton mode, i.e. with $\left|x_{1}-x_{2}\right| \gg \nu / \lambda u$, is a long-lived excitation, hence justifying the heuristic argument.

In order to lend support to the heuristic quasi-particle picture based on the elementary kink solitons (quarks) and the composite pair soliton as the basic quasi-particle it is essential to consider soliton collisions. We have here considered the two symmetric cases: i) the collision of two propagating solitons with a static soliton and ii) the collision of two pair solitons. In both cases the configurations are symmetric and the conservation laws are satisfied at all times including the collision regime.

\section{B. Three soliton collisions}

In the first case two propagating solitons moving in opposite directions collide with a static soliton located at the center. The trial solution has the form

$$
\begin{aligned}
& u(x, t)=-\operatorname{sign}(t)\left[u_{1}(x+v t)+u_{1}(x-v t)-2 u_{1}(2 x)\right] \\
& p(x, t)=-2 \nu\left[u_{1}(x+v t)+u_{1}(x-v t)\right] \text { for } t<0 \\
& p(x, t)=-4 \nu u_{1}(2 x) \text { for } t>0
\end{aligned}
$$

and the height field

$$
\begin{aligned}
& h(x, t)=-\operatorname{sign}(t)\left[\frac{2 \nu}{\lambda}\right] \times \\
& \log \left|\frac{\cosh \left[\frac{\lambda|u|}{2 \nu}(x-v t)\right] \cosh \left[\frac{\lambda|u|}{2 \nu}(x+v t)\right]}{\cosh \left[\frac{\lambda|u|}{2 \nu} x\right]}\right|
\end{aligned}
$$

with velocity $v=\lambda u$.

In this mode two left hand solitons with amplitude $2 u$ propagate with equal and opposite velocities toward a static right hand soliton with amplitude $4 u$ located at the center. During the collision the left hand solitons are absorbed, the static right hand soliton flips over to a static left hand soliton and two right hand solitons emerge propagating away from the center with equal and opposite velocity. The solitons thus collide transparently with the static soliton, i.e., there is no reflection, and there is no phase shift associated with the collision. In terms of the 
associated height profile this scattering situation corresponds to filling in a dip with subsequent nucleation of a growing tip.

Energy and momentum are associated with the noiseinduced left hand solitons moving on the noisy manifold $p=2 \nu u$. By inspection of (20) it follows that the total energy $E=-(32 / 3) \nu \lambda u^{3}$, the total momentum $\Pi=0$, and the total area $M=0$ are conserved during the collision.

Choosing the amplitude $u=2$ we have in Fig. 6 depicted the numerical verification of the slope field $u$ as a function of $x$ for different values of $t$. In Figs. 7 and 8 we have shown the associated noise field $p$ and the height profile $h$ as a function of $x$ for the same values of $t$. In Fig. 9 we have shown a gray-scale representation of $u$ in the $x t$ plane. We notice that there is no phase shift associated with the scattering process. Finally, in Fig. 10 we have shown the convergence of energy, momentum, and area during the forward and backward time sweeps integrating numerically the field equations.
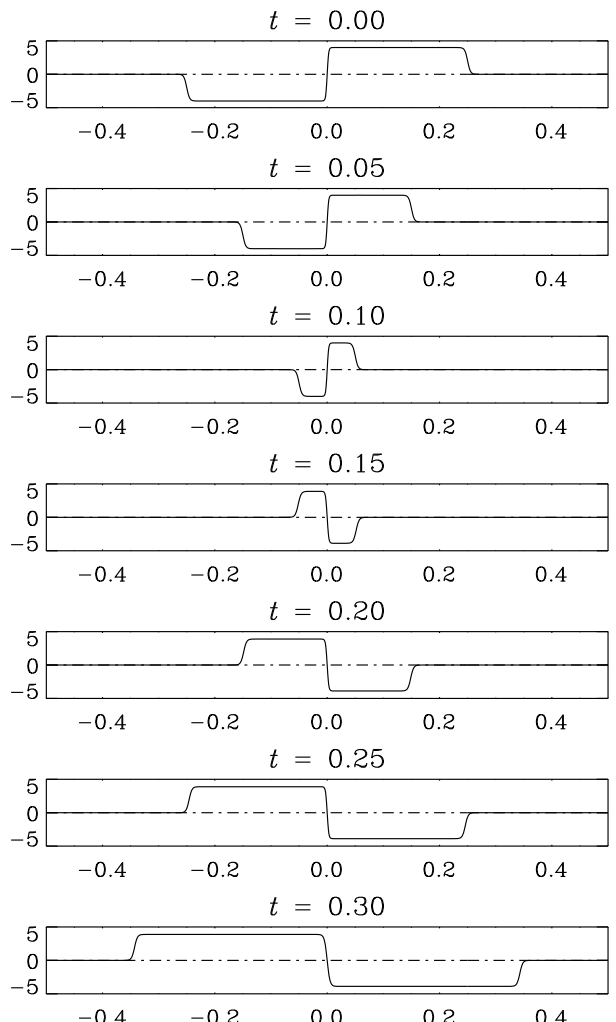

FIG. 6. Three soliton collision: The slope field $u$ as a function of $x$ for different values of $t$.
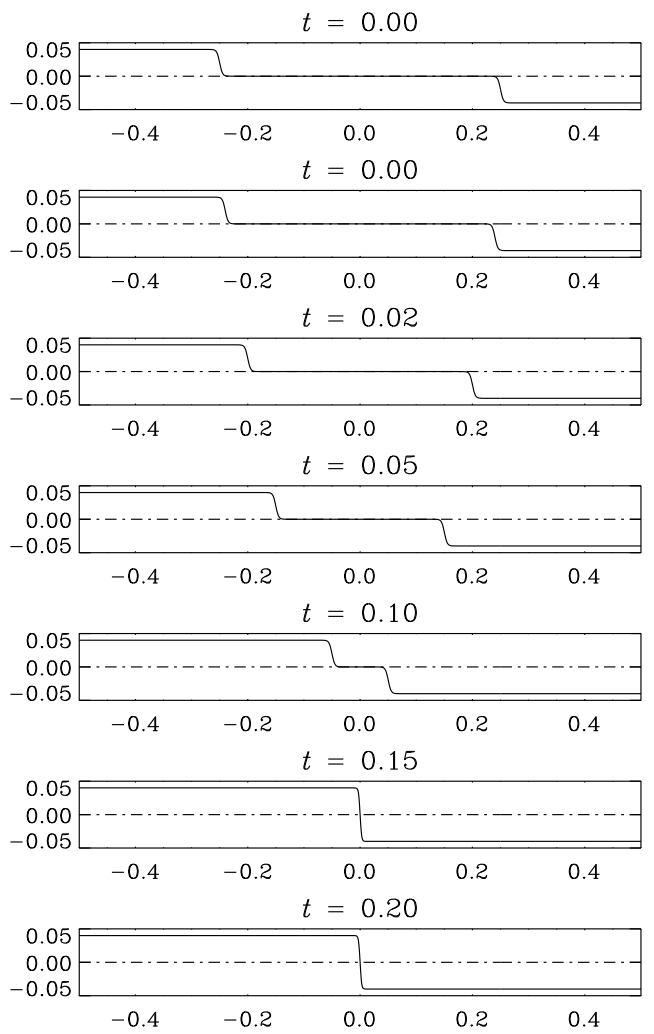

FIG. 7. The noise field $p$ as a function of $x$ for the same values of $t$ as in Fig. 6 .
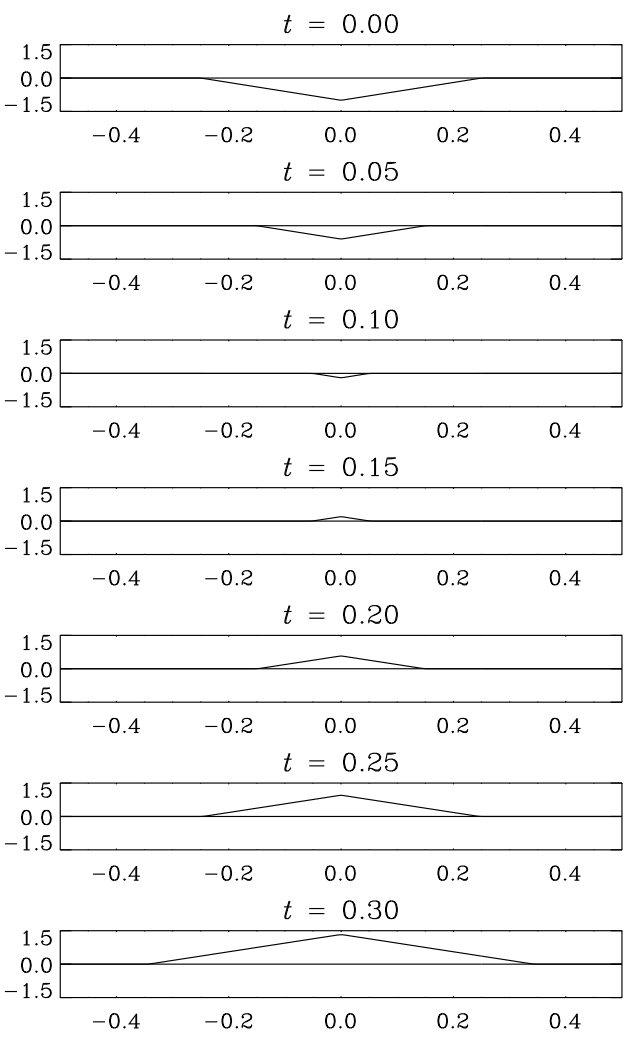

FIG. 8. The height profile $h=\int u \mathrm{~d} x$ as a function of $x$ for the same values of $t$ as in Fig. 6 . 


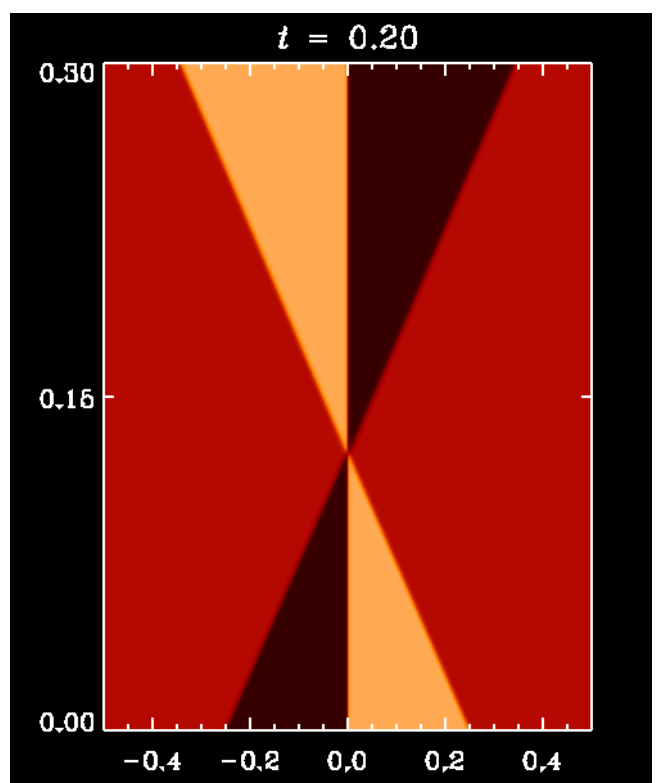

FIG. 9. Gray-scale representation of $u$ in the $x t$ plane showing the three soliton collision. Note the absence of a phase shift dupirag the collision.
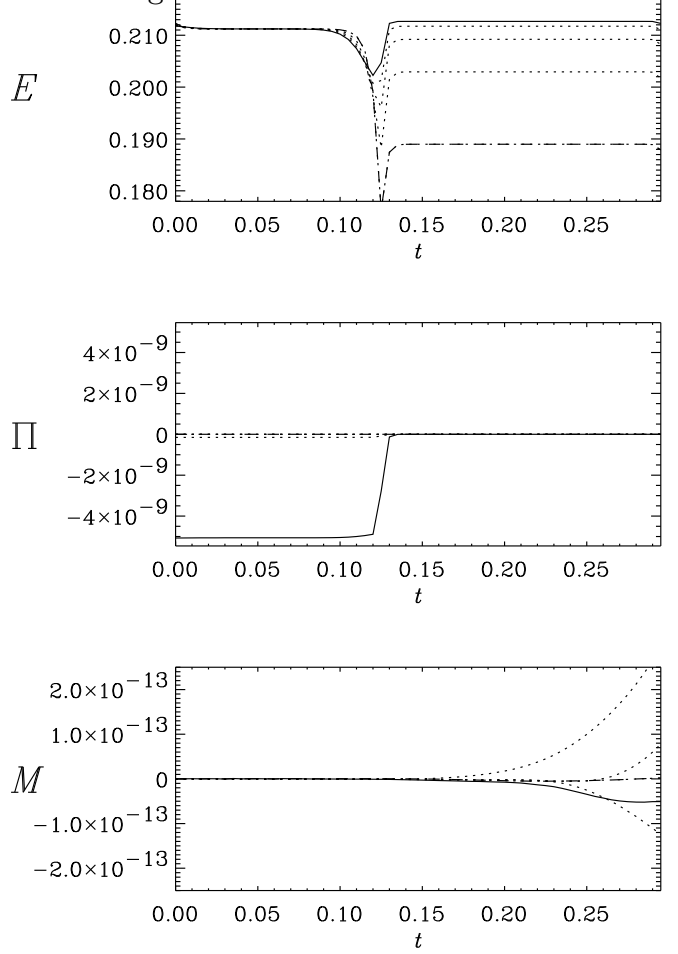

FIG. 10. Evolution of $E, \Pi$, and $M$ during the first few iteration steps. The dashed line indicates the first iteration step and the solid line the last one. Dotted lines are for intermediate steps.

\section{Pair soliton collisions}

In the second case we consider the collision of two pair solitons of equal size and amplitude. The trial solution propagating with velocity $v=\lambda u$ has the form

$$
\begin{aligned}
u(x, t)= & -\left[u_{1}\left(x-v t+x_{1}\right)-u_{1}\left(x-v t+x_{2}\right)\right] \\
& +\left[u_{1}\left(x+v t-x_{2}\right)-u_{1}\left(x+v t-x_{1}\right)\right] \\
p(x, t)= & -2 \nu\left[u_{1}\left(x-v t+x_{1}\right)+u_{1}\left(x+v t-x_{1}\right)\right]
\end{aligned}
$$

for $0<v t<x_{2}$,

$$
\begin{aligned}
u(x, t)= & -\left[u_{1}\left(x-v t+x_{1}\right)-u_{1}(x)\right] \\
& +\left[u_{1}(x)-u_{1}\left(x+v t-x_{1}\right)\right] \\
p(x, t)= & -2 \nu\left[u_{1}\left(x-v t+x_{1}\right)+u_{1}\left(x+v t-x_{1}\right)\right]
\end{aligned}
$$

for $x_{2}<v t<x_{1}$,

$$
\begin{aligned}
u(x, t)= & +\left[u_{1}\left(x+v t-x_{1}\right)-u_{1}(x)\right] \\
& -\left[u_{1}(x)-u_{1}\left(x-v t+x_{1}\right)\right] \\
p(x, t)= & -2 \nu\left[u_{1}(x)+u_{1}(x)\right]
\end{aligned}
$$

for $x_{1}<v t<2 x_{1}-x_{2}$, and

$$
\begin{aligned}
u(x, t)= & +\left[u_{1}\left(x+v t-x_{1}\right)-u_{1}\left(x+v t-2 x_{1}+x_{2}\right)\right] \\
& -\left[u_{1}\left(x-v t+2 x_{1}-x_{2}\right)-u_{1}\left(x-v t+x_{1}\right)\right] \\
p(x, t)= & -2 \nu\left[u_{1}\left(x+v t+2 x_{1}-x_{2}\right)\right. \\
& \left.+u_{1}\left(x-v t+2 x_{1}-x_{2}\right)\right]
\end{aligned}
$$

for $2 x_{1}-x_{2}<v t$.

In this mode two pair solitons of amplitude $2 u$ propagate with equal and opposite velocities toward one another. The two leading kink solitons merge to a static soliton and the two trailing kinks are absorbed. Subsequently, the static right hand soliton flips over to a static left hand soliton and the two pair solitons re-emerge. Analyzing the collision it follows that the scattering of pair solitons is transparent and accompanied by a phase shift in space equal to the soliton size $\left|x_{2}-x_{1}\right|$ or, equivalently, a time delay $\left|x_{2}-x_{1}\right| / v$. In terms of the associated height profile the scattering situation corresponds to filling in a trough due to two colliding steps and the subsequent nucleation of a growing plateau.

By inspection it again follows that the total energy $E=-(32 / 3) \nu \lambda u^{3}$, the total momentum $\Pi=0$, and the total area $M=0$ are conserved during collision.

Choosing the amplitude $2 u$ and the kink positions $x_{1}=0.25$ and $x_{2}=0.15$ we have shown in Fig. 11 the numerical verification of the slope field $u$ as a function of $x$ for different values of $t$. In Figs. 12 and 13 we have shown the associated noise field $p$ and the height profile $h$ as a function of $x$ for the same values of $t$. In Fig. 14 we have shown a gray-scale representation of $u$ in the $x t$ plane. We notice the phase shift engendered during the transparent collision. Finally, in Fig. 15 we have shown the convergence of energy, momentum, and area during the forward and backward time sweeps in the numerical integration. 

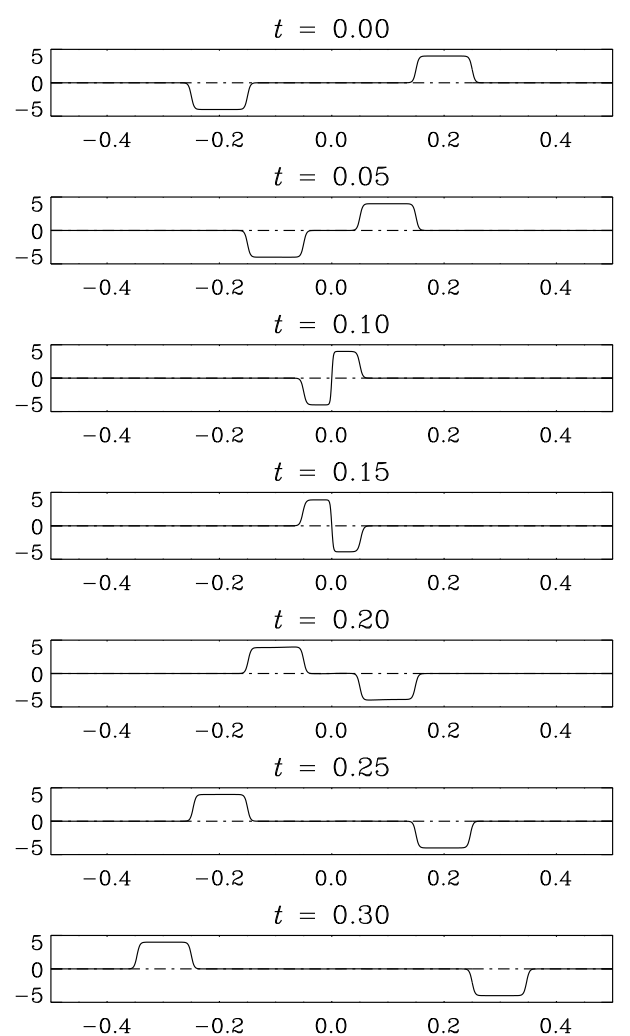

FIG. 11. Pair soliton collision: The slope field $u$ as a function of $x$ for different values of $t$.

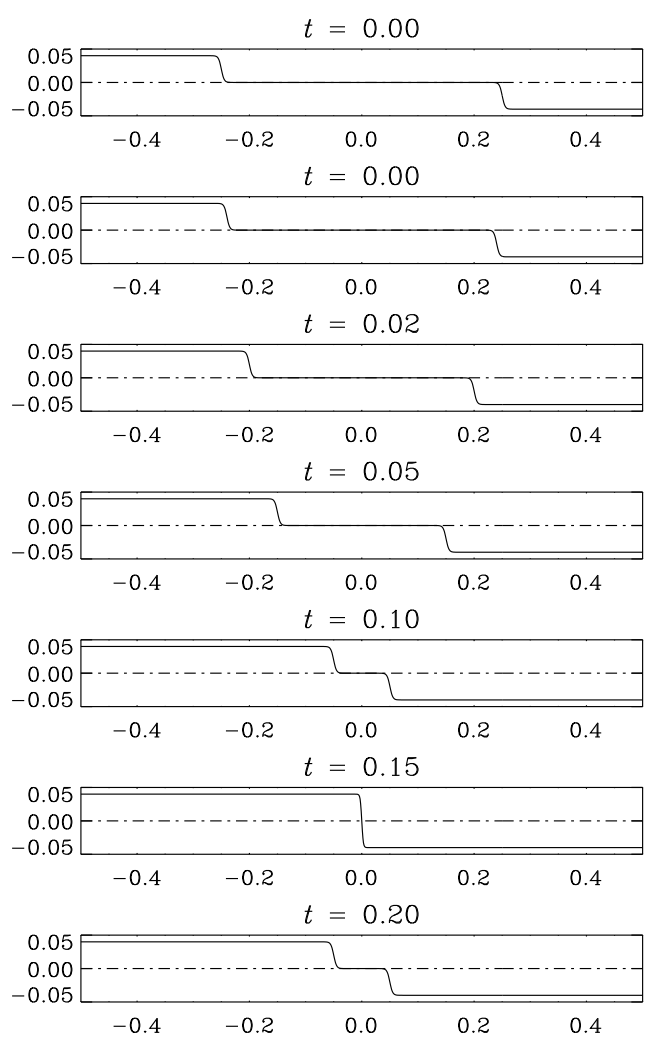

FIG. 12. The noise field $p$ as a function of $x$ for the same values of $t$ as in Fig. 11 .
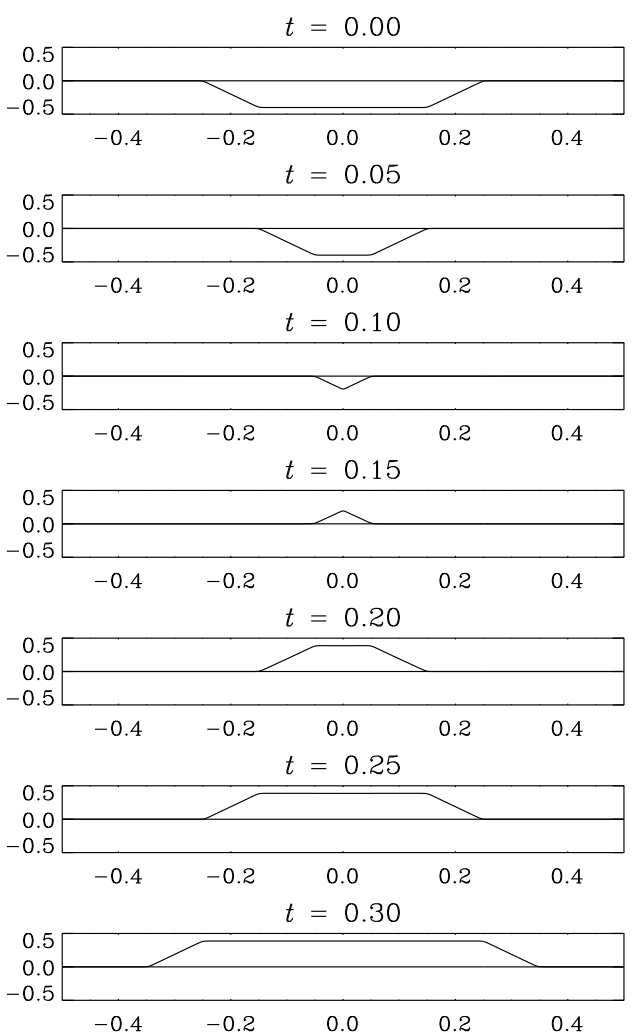

FIG. 13. The height profile $h=\int u \mathrm{~d} x$ as a function of $x$ for the same values of $t$ as in Fig. 11 .

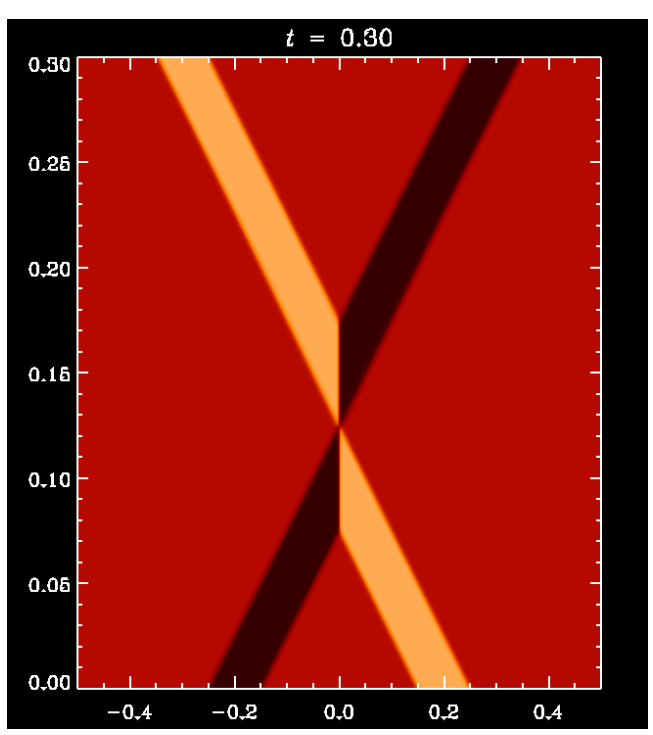

FIG. 14. Gray-scale representation of $u$ in the $x t$ plane showing the pair soliton collision. Note the occurrence of a phase shift during the collision. 

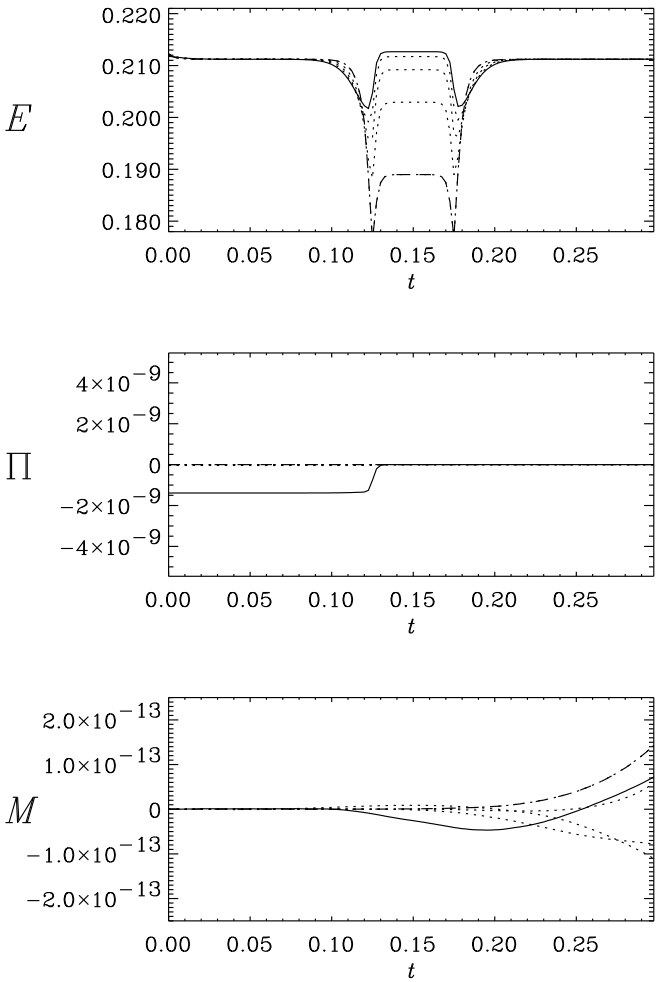

FIG. 15. Conserved quantities depicted as in Fig. 10.

\section{GROWTH AND NUCLEATION}

Since we have achieved numerical justification of three specific dynamical soliton configurations, namely i) the pair soliton, ii) the collision of two solitons with a static soliton, and iii) the collision of two pair solitons, we can proceed to draw some simple conclusions based on the general framework discussed in Sect. 1. There are two levels of description: the stochastic Langevin level and the deterministic Fokker-Planck or equation of motion level. On the Fokker-Planck level yielding the canonical field equations (5) and (5) the growth of the interface is interpreted in terms of a gas of propagating solitons (and diffusive modes). The stochastic description on the Langevin level is then established in the weak noise limit $\Delta \rightarrow 0$ by computing the action $S$ associated with a particular dynamical mode and subsequently deduce the probability distribution according to (9), i.e., $P \propto \exp (-S / \Delta)$. This procedure is completely equivalent to the WKB limit of quantum mechanics. Here the wavefunction $\Phi$ and thus the probabilistic interpretation is given by $\Phi \propto \exp (i S / \hbar)$, where $S$ is the action associated with the classical motion [26]. Note that unlike quantum mechanics there is no phase interference in the stochastic nonequilibrium case.

\section{A. The pair soliton: anomalous diffusion}

The long-lived pair soliton (17) has size $\ell=\left|x_{1}-x_{2}\right|$, amplitude $2 u$, and propagates with velocity $v=-\lambda u$. During a revolution in a system of size $L$ with periodic boundary conditions the height field increases with a layer of thickness $2 u \ell$. Since the system is traversed in time $t=L / v$ the integrated growth velocity is given by $2 \lambda u^{2} \ell / L$ which for a single pair of fixed size vanishes in the thermodynamic limit. On the other hand,the local growth velocity $d h / d t$ is given by $2 \lambda u^{2}=(\lambda / 2)(\nabla h)^{2}$ which is consistent with the averaged $\mathrm{KPZ}$ equation (3) in the stationary state.

The stochastic properties of the pair soliton growth mode is also easily elucidated by noting that the action associated with the pair mode is given by $S=$ $(4 / 3) \nu \lambda|u|^{3} t$. Denoting the center of mass of the pair mode by $x=\left(x_{1}+x_{2}\right) / 2$ we have $u=v / \lambda=x / t \lambda$ and we obtain using (9) the transition probability

$$
P(x, t) \propto \exp \left(-\frac{4}{3} \frac{\nu}{\Delta \lambda^{2}} \frac{x^{3}}{t^{2}}\right),
$$

for the 'random walk' of independent pair solitons or steps in the height profile. Comparing (32) with the distribution for 'ordinary' random walk originating from the Langevin equation $d x / d t=\eta,\langle\eta \eta\rangle(t)=\Delta \delta(t)$, $P(x, t) \propto \exp \left(-x^{2} / 2 \Delta t\right)$, we observe that the growth mode performs anomalous diffusion. The distribution (32) also implies the soliton mean square displacement, assuming pairs of the same average size,

$$
\left\langle x^{2}\right\rangle(t) \propto\left(\frac{\Delta \lambda^{2}}{\nu}\right)^{1 / z} t^{2 / z}
$$

with dynamic exponent $z=3 / 2$, identical to the dynamic exponent defining the KPZ universality class. This result should be contrasted with the mean square displacement $\left\langle x^{2}\right\rangle \propto \Delta t^{2 / z}, z=2$, for ordinary random walk. The growth modes thus perform superdiffusion.

\section{B. Soliton collisions: nucleation}

The three soliton scattering case shown in Figs. 6, 7, and 8 corresponds to the filling in of a dip and the subsequent nucleation of a tip. The incoming solitons have amplitude $2 u$ and move with velocities $v= \pm \lambda u$. Denoting the distance of a soliton from the center by $d$, the height change from the bottom of the dip to the top of the tip is $\Delta h=4 u d$. The duration of the collision process is $\Delta t=2 d / v$ and we obtain for the local growth velocity $\Delta h / \Delta t=(\lambda / 2)(2 u)^{2}=(\lambda / 2)(\nabla h)^{2}$, consistent with the $\mathrm{KPZ}$ equation.

As regards the stochastic properties the action prior to the collision is associated with the incoming left hand solitons and given by $(8 / 3) \nu \lambda u^{3} t$. After the collision the static left hand soliton of amplitude $4 u$ carries the action 
$(32 / 3) \nu \lambda u^{3} t$. There is thus an increase of action in connection with the tip formation yielding, according to (9), a reduced probability, i.e., a statistical suppression of tip nucleation.

The case of pair soliton scattering shown in Figs. 11, 12 , and 13 correspond to the filling in of a trough and the subsequent formation of a growing plateau. The incoming pair solitons have amplitude $2 u$ and propagate with velocities $v= \pm \lambda u$. Denoting the pair size by $d$ the height change from the trough to the plateau is $\Delta h=4 u d$, the duration of the collision $\Delta t=2 d / v$, and we obtain again $\Delta h / \Delta t=(\lambda / 2)(\nabla h)$, consistent with the KPZ equation.

Before and after the collision the action is now unchanged and equal to $(8 / 3) \nu \lambda u^{3} t$. As in the three soliton case this action comes from the two left hand solitons. However, during the collision in a time interval $\Delta t=d / v$ the action jumps to the value $(32 / 3) \nu \lambda u^{3} t$ as the static left hand soliton of amplitude $4 u$ is temporarily formed before the re-emergence of the pair solitons. Comparing this situation with the tip formation in the three soliton scattering case we conclude that tip formation is statistically suppressed with respect to the formation of a growing plateau. Generally the formation of tips is 'expensive' since a large change in slope yields a large change in action.

\section{SUMMARY AND CONCLUSION}

In the present paper we have numerically investigated the coupled diffusion-advection type field equation originating from the canonical phase space approach applied to the noisy Burgers equation or the equivalent KPZ equation in one spatial dimension. We have shown that the pair soliton mode in the slope field corresponding to moving steps in the height field forms a long-lived excitation. We have furthermore investigated two special scattering scenarios, namely the collision of two identical moving solitons with a static soliton and the collision of two identical pair solitons. They correspond in the height field respectively to the nucleation of a growing tip and to the formation of a growing plateau. Finally, we have applied the canonical phase space approach in order to estimate the stochastic aspects of the above configurations and found i) that a step in the height field performs a random walk with dynamical exponent $z=3 / 2$ corresponding to averaged superdiffusion and ii) that tip formation is stochastically suppressed with respect to plateau formation.

As discussed above the inherently unstable structure of the field equation makes a direct integration forward in time inaccessible and we can thus not establish solutions as an initial value problem and discuss the equations generically. Consequently, we are limited to numerically check trial solutions representing a variety of scattering situations. So far we have only been able to verify the symmetric cases of three soliton and soliton pair collisions. In order to extend the present numerical approach and thus provide substance to the heuristic quasi-particle representation of a growing interface it is clearly of interest to design more involved trial solutions. Alternatively, a completely different approach to generate solutions is called for.

[1] D. Forster, D. R. Nelson, and M. J. Stephen, Phys. Rev. Lett. 36, 867 (1976).

[2] D. Forster, D. R. Nelson, and M. J. Stephen, Phys. Rev. A 16, 732 (1977).

[3] M. Kardar, G. Parisi, and Y. C. Zhang, Phys. Rev. Lett. 56, 889 (1986).

[4] E. Medina, T. Hwa, M. Kardar, and Y. C. Zhang, Phys. Rev. A 39, 3053 (1989).

[5] T. Halpin-Healy and Y. C. Zhang, Phys. Rep. 254, 215 (1995).

[6] A. L. Barabasi and H. E. Stanley, Fractal Concepts in Surface Growth (Cambridge University Press, Cambridge, 1995).

[7] J. Krug, Adv. Phys. 46, 139 (1997).

[8] E. Frey and U. C. Täuber, Phys. Rev. E 50, 1024 (1994).

[9] E. Frey, U. C. Täuber, and T. Hwa, Phys. Rev. E 53, 4424 (1996).

[10] E. Frey, U. C. Täuber, and H. K. Janssen, Europhys. Lett 47, 14 (1999).

[11] H. Janssen, U. Täuber, and E. Frey, J .Eur. Phys. B 9, 491 (1999).

[12] M. Lässig, Phys. Rev. Lett. 80, 2366 (1998).

[13] M. Lässig, Phys. Rev. Lett. 84, 2618 (2000).

[14] F. Colaiori and M. A. Moore, cond-mat/0010410.

[15] F. Colaiori and M. A. Moore, cond-mat/0011191.

[16] P.C.Martin, E.D.Siggia, and H.A.Rose, Phys. Rev. A 8, 423 (1973).

[17] R.Baussch, H.K.Janssen, and H.Wagner, Z. Phys. B 24, 113 (1976).

[18] H. C. Fogedby, Phys. Rev. E 57, 4943 (1998).

[19] H. C. Fogedby, Phys. Rev. Lett. 80, 1126 (1998).

[20] H. C. Fogedby, A. B. Eriksson, and L. V. Mikheev, Phys. Rev. Lett. 75, 1883 (1995). 
[21] M. I. Freidlin and A. D. Wentzel, Random Perturbations of Dynamical Systems (Springer-Verlag, New York, 1984).

[22] R. Graham and T. Tél, J. Stat. Phys. 35, 729 (1984).

[23] R. Graham, Noise in nonlinear dynamical systems, Vol 1, Theory of continuous Fokker-Planck systems, eds. F. Moss and P. E. V. McClintock (Cambridge University Press, Cambridge, 1989).

[24] H. C. Fogedby, Phys. Rev. E 59, 5065 (1999).

[25] H. C. Fogedby, Phys. Rev. E 60, 4950 (1999).

[26] L. Landau and E. Lifshitz, Quantum Mechanics (Pergamon Press, Oxford, 1959).

[27] L. Landau and E. Lifshitz, Mechanics (Pergamon Press, Oxford, 1959).

[28] S. F. Edwards and D. R. Wilkinson, Proc. Roy. Soc. London A 381, 17 (1982).

[29] D. A. Huse, C. L. Henley, and D. S. Fisher, Phys. Rev. Lett. 55, 2924 (1985).

[30] H. C. Fogedby, cond-mat/0005182.

[31] H. C. Fogedby, Phys. Rev. E 57, 2331 (1998).

[32] F. J. Sánchez-Salcedo and A. Brandenburg, Month. Not. Roy. Proc. Soc. 322, 67 (2001). 